\title{
NARRATIVAS VITALES EN LAS SOCIALIDADES DEL CURRÍCULO UNIVERSITARIO PARA LA FORMACIÓN INICIAL DEL PROFESORADO
}

\author{
MARÍA CRISTINA SARASA
}

Universidad Nacional de Mar del Plata

RESUMEN

Este trabajo plantea una indagación narrativa sobre las socialidades del currículo universitario, donde confluyen el entorno social y las vidas de los estudiantes. Su contexto específico es la formación inicial del profesorado de inglés en una universidad nacional argentina. El marco teórico define al currículo como una construcción social donde se negocian significados, conceptualizando las nociones del currículo formal y del currículo de vidas. El diseño e implementación del estudio se inscriben en la indagación narrativa como metodología de investigación. Ésta aborda principalmente el entramado de naraciones estudiantiles respecto de las socialidades curriculares que habitan relatos de vida compuestos por veinticuatro estudiantes de grado de un profesorado de inglés universitario. Las tematizaciones narrativas emergentes develan a los co-autores y co-protagonistas de los currículos experienciales-vitales y educativos de estos participantes. El análisis de estas tramas relatadas posee implicancias en torno al diseño, la reforma y/o la implementación de planes de estudio para el profesorado universitario. En este contexto, el trabajo aporta al diálogo sobre la valoración pública de lo privado en los procesos curriculares, sosteniendo un compromiso ético con dimensiones medulares, a la hora de pensar, negociar y enseñar el currículo universitario para la formación docente.

Palabras clave: Educación superior. Currículo. Formación del profesorado. Socialidades. Indagación narrativa.

\section{ABSTRACT LIFE NARRATIVES IN THE SOCIALITIES OF THE UNIVERSITY CURRICULUM FOR INITIAL TEACHER EDUCATION}

This paper constitutes a narrative inquiry into the socialities of university curriculum, lying at the intersection of students' lives and social milieus. The research context is an English teacher education program at an Argentinean state university. Its rationale defines curriculum as a 
social construction where meanings are negotiated and conceptualizes the notions of formal curriculum and curriculum of lives. Its design and implementation adopt narrative inquiry as research methodology. It mainly addresses the emplotment of students' narratives about curriculum socialities present in life stories composed by twenty-four undergraduates of an English teacher education program. Emergent narrative thematizations disclose co-authors and co-protagonists in participants' life experiential and educational curricula. Analyzing these emplotted experiences bears implications for the design, reform, and/or implementation of curricula for university teacher education. In this context, this work contributes to the conversation on the public worth of the private in curricular processes, building and sustain and ethical commitment with core dimensions for conceiving, negotiating, and communicating university curricula for teacher education.

Keywords: Higher education. Curriculum. Teacher education. Socialities. Narrative inquiry.

RESUMO NARRATIVAS VITAIS NAS SOCIALIDADES DO

\section{CURRÍCULO UNIVERSITÁRIO PARA A FORMAÇÃO DE PROFESSORES}

Este trabalho expõe uma pesquisa narrativa sobre as socialidades do currículo universitário, que confluem o meio social e as vidas dos estudantes. O contexto específico é a formação inicial da Licenciatura de inglês, em uma universidade nacional argentina. O marco teórico define o currículo como uma construção social, onde se negociam significados, conceitualizando as noções de currículo formal e currículo de vidas. o esquema e a implementação do trabalho se inscrevem na pesquisa narrativa como metodologia de investigação. Esta aborda principalmente a rede das narrações estudantis a respeito das socialidades curriculares que habitam nos relatos de vida formados por vinte e quatro estudantes de determinada licenciatura de inglês universitário. As tematizações narrativas resultantes desvelam aos coautores e aos coprotagonistas dos currículos experiências vitais e educativas destes participantes. A análise destas tramas relatadas possui implicâncias em torno do desenho, reforma e implementação de programas de estudo para a Licenciatura universitária. Neste contexto, o trabalho subsidia o diálogo sobre a valoração pública do privado nos processos curriculares, sustentando um compromisso ético com dimensões medulares, na hora de pensar, negociar e ensinar o currículo universitário para a formação docente. Palavras-chave: Educação superior. Currículo. Formação de professores. Socialidades. Pesquisa narrativa. 


\section{Introducción}

Este trabajo presenta una indagación narrativa (narrative inquiry, CLANDININ, 2013) en torno al significado de las experiencias vividas (DEWEY, 1929/1925) en las socialidades culturales e institucionales que forman el currículo universitario (CAINE y STEEVES, 2009), donde confluyen diversos entornos sociales, así como las vidas de los estudiantes. El contexto específico de nuestra investigación es la formación inicial del profesorado de inglés en una universidad nacional argentina situada en la costa de la Provincia de Buenos Aires. Nuestro marco teórico aborda al currículo de la formación docente universitaria como una construcción social, un texto político donde transcurren procesos de negociación de significados por parte de sus variados actores (TAYLOR y BOVILL, 2017). En ese sentido, definimos, por una parte, un currículo académico formal (DE ALBA, 1998) y, por otra, un currículo experiencial de vidas donde se entrelazan las existencias de todos sus actores (HUBER ET AL, 2014b). A partir de estas conceptualizaciones, diseñamos nuestra investigación utilizando la metodología de la indagación narrativa (CLANDININ, 2013), abordando tramas de diversas socialidades curriculares tal como fueron contadas en diversos relatos vitales (MCADAMS, 2008), narrados oralmente o escritos por veinticuatro estudiantes de grado del profesorado de inglés en una universidad nacional argentina. La tematización narrativa (CRESWELL, 2012) de esas historias explora el interrogante principal de esta investigación en términos de: ¿cómo se entraman las narraciones de los estudiantes respecto de las socialidades curriculares que habitan sus relatos? En esta pregunta se origina nuestra meta principal, la cual es desentrañar las complejidades de las socialidades curriculares en los entramados de estos relatos estudiantiles.
Nuestro trabajo comienza con un marco teórico sobre el currículo definido éste, como ya indicamos, en términos de una construcción social (FRASER y BOSANQUET, 2006). Luego, transparentamos el proceso, contexto y participantes de la indagación narrativa (CONNELLY y CLANDININ, 2006) llevada a cabo en el profesorado de inglés de una universidad nacional ubicada en la costa de la Provincia de Buenos Aires, Argentina. Las tematizaciones narrativas emergentes de nuestro análisis revelan a los co-autores y co-protagonistas de, primero, el currículo de las vidas de los participantes y, segundo, de sus currículos educativos formales. Seguidamente, retomando la pregunta de la investigación, e intentando dilucidar los entramados narrativos en torno a esos currículos, examinamos las posibles implicancias de estos dos currículos para el diseño, la reforma y/o la implementación de los tradicionalmente denominados currículos prescritos (o planes de estudio) para la formación inicial docente universitaria. Concluimos nuestro trabajo discurriendo sobre sus aportes al diálogo sobre la valoración pública de lo privado, que también sostiene un compromiso ético con dimensiones cardinales, aunque poco señaladas, al momento de pensar, diseñar/reformar, negociar y enseñar el currículo universitario para la formación inicial docente en nuestro país.

\section{Marco teórico}

Nuestro marco teórico se inscribe en la noción del currículo (en este caso de la formación inicial docente universitaria) en términos de la construcción social de un texto político, es decir público, donde se imbrican procesos sociales y personales de negociación de significados (TAYLOR y BOVILL, 2017). Se trata de "una práctica situada y social existente entre relaciones estructurales y actores individuales" (HÖKKÄ, 
ETELÄPELTO y RASKU-PUTTONEN, 2010, p. 3). Visualizamos al currículo no como producto - la estructura y contenido de una materia o de una carrera - sino como al "proceso dinámico e interactivo de enseñar y aprender" (FRASER y BOSANQUET, 2006, p. 272) donde la continuidad de las experiencias (DEWEY, 1929/1925) de aprendizaje de los estudiantes se despliega cotidianamente en el mundo y no exclusivamente en las aulas (SANDLIN, SCHULTZ y BURDICK, 2010).

La presencia de la experiencia en el campo del currículo universitario para la formación docente - definido éste procesualmente sugiere desde hace ya tiempo que "ignorar lo que el futuro docente conoce y ha experimentado, lo que ese docente aspira ser y lograr, es sellar completamente una mina de oro frente a la pobreza" (SARASON, 1993, p. 150). Otros autores comparten la metáfora, sosteniendo que los estudiantes necesitan "guías que les muestren cómo caminar en vez de instructores que se aseguren de que tomen un solo camino, ya abarrotado" para así "ahondar en las profundidades de su carácter y personalidad, donde presumiblemente yacen sus ricos depósitos" (BAUMAN, 2009, p. 162) o sus fondos familiares y privados, es decir sus propias "reservas de conocimiento" (GROSSMAN y MCDONALD, 2008, p. 191).

De esta manera, podemos distinguir, en primer lugar, los procesos de construcción del currículo formal, es decir de "una propuesta político-educativa pensada e impulsada por diversos grupos y sectores sociales cuyos intereses son diversos y contradictorios" (DE ALBA, 1998, p. 59-60). En segundo lugar, destacamos los procesos de edificación del currículo de vidas (curriculum vitae) como un itinerario, un viaje o un transcurso, de carácter vital experiencial, que tiene en cuenta las existencias relatadas de docentes y estudiantes (HUBER ET AL, 2014b). Un currículo de vidas es también un currículo vivido, formado por docentes y estudiantes y a la vez formador de éstos, en una intersección entre sus existencias y sus conocimientos (HUBER ET AL, 2014a). Aquí, los yacimientos individuales y los fondos de conocimiento forman la dotación única de atributos de cada persona, en tanto ser conocedor, que habilita a su singularidad a participar de la generalidad del mundo y así tornar significativas a sus experiencias individuales al compartirlas.

En la investigación sobre la formación del profesorado, la naturaleza de la indagación narrativa (narrative inquiry), como ontología y epistemología (CLANDININ y MURPHY, 2009), radica en su comprensión de la articulación de la experiencia (DEWEY, 1929/1925) manifestada en las existencias relatadas que viven las personas. Abogar, entonces, por el uso de la narrativa en la investigación educativa implica pensar que todos los humanos son narradores que viven esas existencias relatadas donde son, a la vez, co-autores y co-protagonistas. Aquí, la experiencia deviene una categoría ontológica fundamental en términos de la 'realidad' más próxima constituida en la inmediatez de la narrativa. En consecuencia, la indagación narrativa abarca tres 'lugares comunes' de la experiencia que resultan cardinales para tematizar nuestras narrativas. Éstos comprenden su temporalidad, que involucra la continuidad pasado-presente-futuro; su socialidad, que implica la confluencia de los aspectos sociales del entorno y los personales de las vidas interiores; y su localidad, es decir su carácter local y situado (CLANDININ, 2013). Debido a limitaciones editoriales, nuestro trabajo se concentra exclusivamente en las socialidades, focalizando en los entrecruzamientos inter- e intra-personales; públicos y privados; internos y externos y personales y sociales, culturales e institucionales (HUBER ET AL, 2014a). De esta forma, abordamos variados co-protagonistas y co-autores de los currículos de las vidas y de los currículos formales (escolares y universita- 
rios) de los participantes de esta indagación narrativa.

Para pensar y negociar el currículo de la formación inicial del profesorado, recuperamos la noción de que la práctica docente es "idiosincrática" y constituye la "expresión de una manera personal y profesional de conocer fundada en eventos y experiencias del pasado y del presente que ocurren en el hogar, la escuela y en esferas sociales y políticas más amplias" (COLE y KNOWLES, 1993, p. 474). Desde hace ya tiempo, sabemos que los relatos constituyen formas poderosas para explorar el conocimiento práctico personal de los (futuros) profesores. Éste “reside en la experiencia pasada del docente, en su mente y cuerpo actuales y en sus acciones y planes futuros". Se trata de "su manera particular de reconstruir el pasado y las intenciones futuras para enfrentar las exigencias de la situación presente" (CONNELLY y CLANDININ, 1988, p. 25).

Emprender el diseño, la reforma o la enseñanza de los currículos de la formación docente implica relatar y escuchar historias, fundando un compromiso con procesos de indagación narrativa reflexiva y prácticas educativas auténticas. De esta manera, entendemos también hace tiempo que "aprender sobre la enseñanza deviene un proceso autobiográfico" donde el currículo y la didáctica abarcan "los relatos personales y la indagación reflexiva". Los relatos no constituyen una mera "herramienta o [...] una estrategia" sino que encarnan "una orientación, una perspectiva, una práctica" (COLE, 2003, p. 5) que pusimos en marcha con el diseño de nuestra indagación narrativa.

\section{Diseño y participantes de la investigación}

Como metodología cualitativa de investigación (DENZIN y LINCOLN, 2005), la indagación narrativa (narrative inquiry, CLANDININ, 2013) aborda ontológica y epistemológicamente a la "experiencia como relato" (CONNELLY y CLANDININ, 2006, p. 375). En otras palabras, la indagación narrativa implica contar y volver a contar experiencias, recuperándolas como fenómenos de nuestro estudio.

La autora de esta indagación narrativa forma parte de su contexto de investigación en una universidad nacional argentina, donde ejerce funciones de docentes-investigadores universitarios. Esta 'posicionalidad' requiere dos dimensiones éticas para con este trabajo (GUILLEMIN y GILLAM, 2004). La primera es procedimental y demanda la obtención del consentimiento informado de los participantes, así como el mantenimiento de la confidencialidad y el anonimato. La segunda es vincular (CLANDININ y MURPHY, 2009) y se halla basada en los principios del cuidado, la atención y el compromiso con los estudiantes durante toda la indagación. También exige transparentar la suma de los procedimientos mientras se evitan asimetrías excesivas de poder o la imposición de malestares indebidos.

Este trabajo forma parte de una indagación narrativa más extensa sobre tramas relatadas de identidades docentes en historias vivenciales compuestas por veinticuatro estudiantes de grado durante la cursada de una asignatura cuatrimestral de segundo año del área curricular de habilidades lingüísticas del profesorado de inglés en el ámbito de una universidad nacional argentina, emplazada en una ciudad marítima de la Provincia de Buenos Aires. Seguidamente, como corresponde en una indagación narrativa, los presentamos brevemente, identificados con sus seudónimos escogidos.

1. Ant decidió su nombre por el tradicional cuento infantil de la Hormiguita Viajera. Nació en 1990. Antes de ingresar al Profesorado en 2010 comenzó otra carrera en una institución superior. Poseía una experiencia variada en el aprendiza- 
je del inglés durante un total de ocho años y un nivel elemental de italiano.

2. Cas asumió el sobrenombre de Castiel, un personaje que aparece en la cuarta temporada de la serie televisiva Supernatural (KRIPKE, 2008-2009). Perteneciente a la cohorte nacida en 1993, ingresó en 2012 tras una exposición al idioma durante más de trece años. Nunca concurrió a un instituto.

3. Clara se identificó con el personaje de novela Clarissa Dalloway (WOOLF, 2017). Nació en 1991 e ingresó al Profesorado en 2009. No siendo oriunda de la ciudad, necesitó tiempo para adaptarse a la vida local. Asistió a una escuela privada y aprendió inglés en un instituto durante seis años.

4. Coty es su propio diminutivo. Nació en 1993 e ingresó a la carrera en 2012. Acudió siempre a una escuela parroquial concurriendo durante nueve años al mismo instituto de Tute, quien le ha dado clases.

5. Emma tomó su nombre de la novela homónima de Austen (1964). Integraba el grupo de nacidos en 1990 e ingresantes en 2009. Hizo su escolarización en una escuela privada religiosa. Estudió inglés particular y en institutos durante muchos años.

6. Fátima optó por el nombre de la protagonista de La mano de Fátima (FALCONES, 2010). Nacida en 1980, e ingresante en 2011, estudió otra carrera universitaria por dos años. Luego se casó y vivió en España, donde aprendió inglés durante cinco años. Concurrió a una escuela parroquial en la primaria y a una pública en la escuela media (donde estudió francés).

7. Grian eligió su nombre en irlandés. Nació en 1993 e ingresó en 2012 luego de abandonar otra carrera. Tuvo una escolarización heterogénea, concurriendo a la misma escuela bilingüe alemana que Rose y a otras dos más. Estudió inglés durante doce años, jamás en un instituto. Poseía un nivel intermedio avanzado de alemán y uno elemental de francés.

8. Haven (Refugio) es una adaptación de Heaven (Cielo), personaje de la saga Crepúsculo (MEYER, 2005). Nació en 1994 e integraba la cohorte 2012. Fue a escuelas privadas, asistiendo a uno de los mismos institutos de inglés que Sofia. Su experiencia en los institutos fue de siete años.

9. James Nicholas White fusionó sus nombres con los de Colmillo Blanco (White Fang, LONDON, 2008). Nació en 1990 e ingresó al Profesorado en 2010 tras probar otra carrera. Estudió en una escuela parroquial local. Aprendió inglés en el colegio durante nueve años, sin concurrir a un instituto.

10. Jo eligió un diminutivo de su nombre. Nacida en 1993, asistió a una escuela parroquial y estudió en un instituto durante cuatro años.

11. Juana optó por el nombre de su tía. Nacida en 1990, se trasladó por el país debido al trabajo de su padre. Ingresó al Profesorado en 2012 luego de probar dos carreras. Durante su escolarización cambió muchas veces de instituciones educativas. Estudió inglés durante diez años.

12. Lily escogió a la madre de Harry Potter (ROWLING, 1999). Nacida en 1994, fue ingresante en 2012. Siempre concurrió a una escuela privada laica donde estudió inglés desde el nivel inicial durante trece años. Tampoco asistió nunca a un instituto.

13. Maggie favoreció el nombre de su pe- 
rra. Nació en 1992 e ingresó en 2012. La carrera le ha resultado dificil por su 'base previa' de conocimientos del idioma, adquirida durante cuatro años en un instituto. Asistió a una escuela parroquial y desea a ser intérprete.

14. Marilyn optó por el seudónimo de la actriz estadounidense Marilyn Monroe. Nació en la ciudad en 1992 e ingresó en 2010. La carrera le ha resultado larga también por sus conocimientos previos y sus ocupaciones como princesa de belleza. Estudió en una escuela parroquial y asistió a dos institutos de inglés durante seis años.

15. Polka Dots (Lunares) decidió su sobrenombre por la cubierta a lunares de su carpeta. Nació en 1986 e ingresó en 2006, tras abandonar dos carreras. Fue a una escuela primaria pública, a una secundaria privada y a profesoras particulares de inglés durante diez años.

16. Rose tomó su apelativo de la rosa blanca. Nació 1993 e ingresó en 2012. Fue a una escuela bilingüe español-alemán donde estudió inglés durante siete años. Su nivel de alemán era avanzado, aunque hacía tiempo no lo hablaba.

17. Rusa eligió su apodo familiar porque es rubia de ojos azules. Nació en 1991. Ingresó a la carrera en 2010. Estudió en un colegio parroquial y concurrió a un instituto de inglés durante nueve años.

18. Savannah escogió al personaje de la novela Dear John (SPARKS, 2009). Nació en 1995 y, al igual que Sofía, ingresó en 2013. Fue siempre a una escuela privada laica donde estudió inglés durante nueve años, sin complementar con un instituto.

19. Sheila Tarnosky de Polasia evocó el nombre que le daba su padre en la niñez. Nació a fines de 1990 e ingresó en
2010 luego de intentar en otra facultad. Cambió muchas escuelas mientras se trasladó con su familia. Su contacto previo con el idioma abarcaba trece años y asistió a tres institutos de inglés.

20. Sofía escogió el nombre de su hermana. Nació en Mar del Plata en 1994 e ingresó en 2013 junto con Savannah. Acudió a un colegio parroquial y a dos institutos durante siete años. Uno de ellos fue el mismo que el de Haven.

21. The Married One (El Casado) se casó en 2013 con una compañera. Nació en 1987 e ingresó al Profesorado en 2006 junto con su entonces novia. Ha invertido tiempo y esfuerzos para casarse y proseguir estudiando. Concurrió a tres escuelas religiosas, incluyendo una técnica. Estudió inglés intermitentemente en varios institutos.

22. Tute eligió su diminutivo genuino. Nació en 1985 e ingresó al Profesorado en 2004. Realizó las cursadas con lentitud mientras empezó a trabajar.

23. Unnamed (Sin Nombre) nació a comienzos de 1991 e inició el Profesorado en 2009. Concurrió siempre a escuelas públicas donde aprendió inglés paralelamente con un instituto durante nueve años. Tenía un dominio avanzado del italiano.

24. Victoria se presentó con uno de sus nombres. Nació en 1985. En 2003 ingresó simultáneamente al profesorado y a otra carrera. Estudió en la escuela privada religiosa de Emma y concurrió a un instituto durante nueve años. Declaraba un nivel pre-intermedio de italiano y uno elemental de francés.

Para recoger las narrativas de los participantes en el aula - durante la cursada de la materia del área de habilidades lingüísticas (re)diseñamos instrumentos y procedimientos 
de recolección de relatos experienciales y de vida en diferentes medios - oral presencial, escrito impreso y virtual. Los temas de estas narrativas escritas y orales se basaron en los utilizados en entrevistas de vida (MCADAMS, 2008) y versaban sobre: (1) los relatos que leemos, miramos y escuchamos (BRITZMAN; PITT, 1996); (2) biografias escolares, universitarias y lingüísticas (DERRIDA, 1996); (3) profesores memorables y héroes de la vida real (MCADAMS, 1996); (4) grandes desafíos superados (MCADAMS, HANEK, y DADABO, 2013); (5) ensayos identitarios (POPE, 1995); (6) caja de la memoria con objetos que representan momentos decisivos (CLANDININ, STEEVES, y CHUNG, 2008); (7) comunicaciones diversas entre investigadores y participantes.

El análisis de los relatos fue primero enteramente narrativo, mediante procesos de co-composición individuales con cada participante y su validación de las narrativas personales que íbamos trazando en conjunto (CRESWELL, 2012). De esta forma, co-escribimos primero relatos biográficos e identitarios (NORTON, 2013) para cada estudiante, entramando todos los textos recogidos. Esto permitió tematizar (VAN MANEN, 2003) muy densamente narrativas grupales alrededor de los tres lugares comunes de la indagación narrativa, la localidad, la socialidad y la temporalidad (CAINE y STEEVES, 2009; CLANDININ, 2013). Para esto, confluyen todas las voces de los estudiantes en un marco coral (BRITZMAN, 2003). Dichos temas surgen de la co-composición y análisis de las narrativas estudiantiles realizadas, tanto individuales como grupales. Las tematizaciones narrativas resultan, entonces de la interacción interpretativa de la autora con los textos relatados por los estudiantes. Esta co-composición de historias y la negociación de puntos de vista y significados (CORTAZZI, 2001) se apoyan en la cristalización, que "combina simetría y sustancia con una variedad infinita de formas, sustancias, trasmutaciones, multi dimensiones y ángulos de enfoque" (RICHARDSON y ST. PIERRE, 2005, p. 963) entre investigadores, participantes y referentes de la literatura.

Los variados temas emergentes en los currículos de vida y formales para cada uno de los tres lugares comunes de la indagación narrativa (CAINE y STEEVES, 2009) - acerca de aprender a ser, devenir profesor de inglés revelan problemáticas situadas en el campo local del currículo para la formación inicial del profesorado de inglés. Para este trabajo, debido a los límites de su extensión, seleccionamos temáticas emergentes que comprenden específicamente las socialidades del currículo de vidas y del currículo formal tal como ya los hemos definido. A continuación, mediante una urdimbre narrativa de relatos multivocales y de prismas de los implicados en la investigación - referimos, en esta ocasión, historias sobre los co-autores y co-protagonistas de las socialidades de estos dos currículos.

\section{Tematizaciones narrativas: Los co-autores del currículo de las vidas y del currículo educativo}

En las socialidades (CLANDININ, 2013) donde los participantes profesores habitan variados co-compositores y co-protagonistas (MCADAMS, 2013). Trazamos dos divisiones curriculares. La primera está integrada por los coprotagonistas/coautores del que ya denominamos currículo de las vidas (HUBER ET AL, 2014a, 2014b), quienes aportan sus caudales de conocimiento (BAUMAN, 2009; GROSSMAN y MCDONALD, 2008; SARASON, 1993). Se trata de textos literarios que los estudiantes han apreciado, de sus series o películas favoritas, de la música que los ha acompañado, de personalidades, de familiares y de allegados a los que admiran como héroes. En la segunda división hallamos a los co-protagonistas y co-autores 
del currículo formal (CAMILLONI, 2013). Recuperamos a docentes-actores de las biografias escolares y a los profesores memorables, junto con representaciones de los estudiantes sobre la buena docencia.

\section{Coescritores y coprotagonistas del currículo de las vidas} Literatura, cine, televisión, música

Los textos literarios, así como las películas y las series televisivas, "son una parte fundamental de quiénes somos [...] cuentan mucho sobre nosotros, nuestros sentimientos, nuestras ideas y nuestros sueños" (UNNAMED). Por su parte, Marilyn elige a Martín Fierro (HERNÁNDEZ, 2017), el libro que impactó en su adolescencia cuando su abuela paterna le enseñó a leerlo para el colegio. Grian señala "Continuidad de los parques" (CORTÁZAR, 1971). Maggie rescata a El amor en los tiempos del cólera (GARCÍA MÁRQUEZ, 2003) porque "a los que esperan les suceden cosas buenas". Finalmente, Haven opta por su cuento favorito "A la deriva" (QUIROGA, 1998).

Prosigue el género épico/fantástico con la trilogía El señor de los anillos (TOLKIEN, 2001) que abrió las puertas a la lectura para Tute. A su vez, James expresa que La llíada (HOMERO, 2006) "revela la esencia del hombre". El favorito de Clara, Sheila, Cas y Juana es la saga del personaje Harry Potter (ROWLING, 1999) porque "existe un mundo paralelo que coexiste con el real [...] más allá de nuestra vida ordinaria hay otra llena de cosas que nunca vimos" (SHEILA). Jo atesora una colección de Winnie the Pooh (MILNE, 2008). Por su parte, Rusa y Cas evocan respectivamente al Diario de Ana Frank (FRANK, 1958) y a Los ojos del perro siberiano (SANTA ANA, 1998). La primera leyó el Diario a la edad de Ana y la segunda rescata del texto su alegato contra la discriminación.
Otros participantes escogen novelas abordadas en el Profesorado de inglés. En ese sentido, Expiación (MCEWAN, 2008) fue el primer libro completo del que Coty disfrutó. Polka menciona Cabalgar el viento (ST. CLAIR ROBSON, 1982) por su tratamiento de los indígenas de EEUU. Ant selecciona a la novela de suspenso Perdida (FLYNN, 2013). En otro orden, Victoria alude a Las penas del joven Werther (VON GOETHE, 2011) que leyó de chica. Emma escoge Orgullo y prejuicio (AUSTEN, 2006) de su escritora favorita y Unnamed Retrato del artista adolescente (JOYCE, 2016) “por la precisión con la cual Joyce [...] expresa perfectamente cómo funciona la mente humana". Entre los best-sellers, Savannah prefiere Las ventajas de ser invisible (CHBOSKY, 2013). Sofía indica a El pájaro canta hasta morir (MCCULLOUGH, 1995) por su desgarradora historia de amor. Rose destaca En mil pedazos (FREY, 2006) por su promesa salvadora. Fátima es apasionada de Ken Follett, con su preferido Los pilares de la tierra (FOLLETT, 2017). Lily cuenta acerca la novela de suspenso El cuento número trece (SETTERFIELD, 2007).

En cuanto a las series y a los films, "nuestros valores y creencias se reflejan a menudo en esas historias que más queremos" (CAS). En primer lugar, para Clara y Tute, se hallan las comedias como Friends (CRANE y KAUFFMAN, 1994-2004), acerca de "seis amigos que soportan juntos las adversidades de la vida" (TUTE). Rose prefiere la creación post 11/9 Rescátame (LEARY y TOLAN, 2004-2011). Savannah arraigó a sus cuatro amigas ingresantes al profesorado en torno a su obsesión, Pequeñas mentirosas (SHEPARD, 2010-2016). Jo y Maggie confiesan su respectivo fanatismo por ER Emergencias (CRICHTON, 1984-2009) y Anatomía de Grey (RHIMES, 2005-2017). Marilyn y Ant mencionan a Breaking bad (GILLIGAN, 2008-2013). Cas y Grian prefieren géneros fantásticos: la primera de terror con In the flesh (MITCHELL, 2013-2015) 
y la segunda de ciencia ficción con Doctor Misterio (DAVIES, 2005-2018). James continúa su adhesión al género épico con Espartaco sangre y arena (DEKNIGHT, 2010). Rusa y Juana han visto muchísimas series con sus madres. Seguidamente, la película animada Anastasia (BLUTH y GOLDMAN, 1997) fue el primer contacto de Emma con una historia de la "vida real". Sofia considera que el mensaje de Cómo entrenar a tu dragón (COWELL, 2010) es universal. Entre los films 'de época', Juana escoge a Orgullo y prejuicio (MOGGACH, 2005). Haven indica que La joven Victoria (FELLOWES, 2009) refleja su gusto por "películas situadas en los siglos XVII, XVII y XIX". Prosiguen ganadoras de la Academia: para Rusa Cinema Paradiso (TORNATORE, 1988) y para Sheila La vida es bella (BENIGNI, 1997). Luego, según Unnamed, Toro Salvaje (SCORSESE, 1980) resulta inigualable. Lily destaca el impacto que Forrest Gump (ROTH, 1994) ha tenido en su vida. Tras los Óscares, prosiguen la distopía La isla (TREDWELL-OWEN, 2005) para Married; el thriller psicológico El efecto mariposa (GRUBER y BRESS, 2004) para Coty; el drama deportivo Persiguiendo mavericks (MEENAGHAN, 2012) para Polka; la aventura de ciencia ficción Transformers (ORCI y KURTZMAN, 2007) para Fátima; y la producción paraguaya Novena (COLLAR, 2012) para Victoria.

En cuanto a la música, el vínculo de muchos participantes con el inglés "se halla íntimamente relacionado con [su] amor por la música" (HAVEN). Savannah y Sofía dividen su fanatismo entre la serie Pequeñas mentirosas y el cantante, compositor y guitarrista británico Ed Sheeran, mientras que Jo admira desde chica a Kelly Clarkson, cantante, compositora y actriz estadounidense. A su vez, Marilyn, Rose, Emma, Maggie, Rusa y Coty han escuchado música rock y pop de la generación de sus padres de 1960 y 1970 - sobre todo británica y las dos últimas a Los Beatles - desde la infancia, buscando las letras para memorizarlas, copiándo- las o averiguando palabras en el diccionario, en un proceso cíclico de querer aprender inglés para comprenderlas y cantarlas o aprender muchos aspectos del idioma mediante éstas. Juana compartía la música con amigos; James la combinaba con videojuegos y Victoria la escuchaba en la radio. Cas alude a la música como parte integral de su vida. Tute siempre escucha nuevas producciones y utiliza canciones en sus clases. Grian también canta, aunque su fuerte son la plástica y la escritura. Mientras Unnamed aprendió de musicales en inglés, su afición por la ópera lo llevó a estudiar italiano y canto. El aria "Ponte el vestuario" de la ópera Payasos (LEONCAVALLO, 1892) "representa cómo debemos tratar de enfrentar la vida diaria, especialmente en una carrera como la docencia que se asemeja a la actuación" (UNNAMED). Los estudios de teatro musical de Lily se vinculan con su pasión por el inglés cuando explica que "el escenario pasó a ser mi casa y la música mi lenguaje". El momento más significativo para Haven fue empezar lecciones de canto. Confiesa que "siempre amé estar sobre el escenario y cantar y bailar. El arte corre por mis venas".

\section{Los héroes cotidianos}

Las biografías de los héroes comunes que relataron los estudiantes prefiguran épicas vicarias que les otorgan valores y generan promesas al implicarse dichas figuras en el bienestar y el devenir de generaciones presentes y futuras (MCADAMS, 1996). Coty explica que "cada vez que enfrento dificultades en la vida, pienso en [mi padre], las situaciones que encontró y las maneras en las que reaccionó". Clara y Unnamed eligen a la pareja de sus progenitores. La primera destaca su esfuerzo "en la lucha contra la enfermedad [fatal] de mi hermana y ayudándonos a mis hermanos y a mí es heroico". Unnamed define a un héroe como alguien 
que "no se mide por el tamaño de su fuerza sino por la fuerza de su corazón". Aunque sus padres se quedaron una vez sin trabajo, relata que "no solamente pudieron superar esas circunstancias terribles [...] sino que nos proveyeron a mi hermano y a mí, satisfaciendo cada una de nuestras necesidades básicas, y no tan básicas". Ant escoge a su padre adoptivo porque dice que es "la persona más sabia que conozco". Rusa, Married, Coty, Polka y Jo cuentan sobre la orfandad y las familias divididas de sus padres. Así, explica Rusa, "cuando pienso en un héroe, pienso en una persona que tuvo que superar muchos obstáculos, especialmente una niñez dificil". El padre de Married "es único porque, a pesar de que sufrió mucho y tuvo poca ropa y comida en su juventud, no quiere nada para él". Coty expresa que su padre "no tenía todas las facilidades que me ofrece ahora. No veía mucho a su padre, su familia tenía dificultades financieras y debió encontrar trabajo a una edad precoz". Polka admira a su padre contador porque "ha intentado constantemente enseñar a sus hijas [...] con acciones". El padre de Jo sumó a la precariedad de su niñez un problema de salud crónico. Actualmente, si bien cumplirá su sueño de recibirse de profesor de arte, debe cuidar de su propio padre. Savannah y Sheila destacan los vínculos que las unen a sus padres ya que "estamos verdaderamente conectados en distintos niveles y nos amamos y respetamos [...] de una forma increíble" (SAVANNAH). El padre de Sheila es un médico que le "ha dado muchas lecciones en la vida".

Marilyn, Rose y Lily escogen a sus abuelos. Las abuelas de las dos primeras son mujeres dinámicas, en la mitad de sus setenta años. La pobreza de su niñez, los desórdenes de sus parejas, la soledad y las enfermedades de sus hijos no le impidieron a una recibirse y trabajar de maestra y a la otra seguir la carrera de asistente social. Lily honró a su abuelo, quien ha- bía fallecido hacía poco. Sufrió la pobreza de niño, padeció enfermedades, trabajó de joven y perdió a sus hermanos y a su esposa. Lily manifiesta que "aspiro a ser tan fuerte y optimista como él lo fue y aún ahora que trato de hacer frente a su pérdida me pregunto ¿qué habría hecho yo?". El tío político de Tute proviene "de orígenes desfavorecidos, ha realizado grandes hazañas o superado adversidades".

Cas, Sofia y Juana refieren a allegados. La primera elige a un amigo porque expresa que "veo a los héroes no como perfectos o sobrehumanos sino como gente común que pelea constantemente para ponerse de pie [...]". El novio de Sofía "ha lidiado con experiencias abrumadoras y a los veinte años nunca ha perdido de vista quién es". Juana expresa acerca de su amiga Catherine que "la considero una heroína no [...] por las situaciones que toleró y superó (a pesar de que hacerlo como lo hizo es suficientemente heroico) sino porque la considero una persona notable y única a causa de lo que hace todos los días". Emma explica que su instructor de Taekwondo es "alguien que se va de viaje y demuestra coraje en la adversidad. El viaje al que me refiero es la vida".

Cuando narramos estos relatos, la joven activista pakistaní Malala Yousafzai había devenido la laureada más joven del Nobel de la Paz, por lo que tres participantes la distinguieron. Grian declara que Malala "es una inspiración para las chicas de todo el mundo [...] como profesoras, deberíamos contar su historia a nuestros estudiantes". Maggie añade que "no podía creer cómo el atentado no la detuvo, sino que le dio la fortaleza de pelear más fuerte contra la injusticia". Victoria destaca la juventud de Malala, su entereza para sobrevivir y el poder de su convocatoria. Por su parte, James refirió al longevo diseñador social Jacque Fresco, una persona "que pone el bienestar de los demás delante del suyo [...] alguien que tiene fe en quienes lo rodean y trata de ver lo mejor de 
la gente". Para Fátima, el fallecido cardiólogo argentino René Favaloro "cansado de 'ser un mendigo en su país' [...] abrumado por la corrupción del sistema de salud [...] se comparó con Don Quijote luchando su batalla solitaria contra los gigantes". Haven se explaya sobre el You Tuber conocido por sus seguidores como Markiplier: un "actor, actor vocal, comediante, personalidad de Internet y comentarista de juegos de videos" quien "ha corrido riesgos que yo no he asumido y probablemente no asumiré por miedo de no triunfar [...] eligió hacer lo que ama en lugar de ajustarse a lo que no le gusta".

\section{Coescritores y coprotagonistas del currículo formal}

\section{Docentes memorables del currículo escolar}

Resulta de larga data la idea acerca de que, mediante el currículo oculto - como opuesto al oficial, escrito y prescrito -, las instituciones educativas hacen mucho más que transmitir conocimiento 'abierta' y 'explícitamente'. En su investigación pionera, Jackson (1996/1968) pensó a la educación como un proceso de socialización y le otorgó una dimensión más amplia y crítica, afirmando que el currículo oculto se refiere a las maneras en que los alumnos aprenden a aceptar la negación de sus propios deseos. Asimismo, el conocido noviciado de la observación (LORTIE, 1975) representa el largo período durante el cual los estudiantes observan diariamente a sus docentes. En este contexto, las biografias escolares y universitarias de nuestros participantes fueron co-protagonizadas por muchos buenos docentes.

Sheila rememora a su maestra de inglés y castellano de primer grado. Revela que se trataba de una persona "sensible y amable [...] No le preocupaba tanto que habláramos inglés perfectamente, estaba interesada en ense- ñarnos lecciones morales". Lily y Rusa señalan a dos profesoras de Matemática. Lily explica que la suya era "apasionada" y "hablaba de los números con mucha alegría y un interés [...] profundo [...] resulta muy claro ahora que es uno de mis modelos como profesora". Rusa declara que su profesora "estaba siempre de buen humor, era apasionada [...] me transmitió esa pasión porque ésa fue la primera y única vez que disfruté de aprender Matemática". Su profesor de Química le enseñó a Sheila “que la única manera de triunfar era pensar y decidir si lo que nos enseñan es correcto o no". James valora de su profesor de Física el haberle "enseñado cómo pensar".

Jo distingue a su profesora de Historia y Sociología porque "sabía compartir ese conocimiento. Explicaba cada tema varias veces [...] usaba [...] diferentes estrategias para explicar. También era exigente". Sofia recuerda a su profesor de Historia que enseñaba Sociología. Indica que "admiro plenamente su fortaleza, su compromiso y su pasión por su trabajo". Ant prosigue con su docente de Historia y señala que "me empezó a gustar la historia y empecé a entenderla. Comprendí que no había una manera definitiva de enseñar [...] los docentes son muy ingeniosos y a veces no se dan cuenta de todas las herramientas que tienen a mano". Siguen una profesora de Historia y una de Literatura de Fátima. Cuenta que "tuvieron un gran impacto en mi vida. Las recuerdo con amor y me gustaría agradecerles por su trabajo duro, su dedicación y el amor que pusieron en sus clases". Unnamed incluye a su profesor de Literatura: "un profesional destacado en su área [...] Su estilo era bastante teatral porque parecía representar una obra corta cada vez que explicaba algo". Rose habla de la arquitecta y profesora de Estética que les enseñaba teoría relacionada con la vida real, incitándolos a dibujar y mostrando sus producciones y fotos. Married aludió a su memorable cuatro veces y 
declaró que "soy un bicho raro porque este profesor que a mí me encantó en la secundaria [...] Éramos los reclutas [...] también lo tuve como el ejemplo de mi viejo, siempre una persona muy disciplinar". Fuera de la escuela, Grian escoge a su mentor de arte y explica que "ha habido un gran debate sobre lo que hace 'grande' a un docente. Algunos dicen el conocimiento, otros dicen la pasión, pero yo creo que es la humildad". Haven elige a su maestro de canto pues indica que "es capaz de sacar a relucir lo mejor de cualquiera y es la prueba viviente de que los sueños sí se hacen realidad si uno trabaja suficientemente duro [...] Por él sigo en el profesorado". Emma refiere a su instructor de Taekwondo en los siguientes términos: "las cosas que él te va diciendo las podés aplicar en [...] el trabajo, en la vida, en la Facultad".

Tres 'docentes' notables habitan el currículo de la vida. La madre de Polka le "ha enseñado [a sus hijas] algunas de las cosas más importantes". Victoria explica que "mi familia ha sido un factor crucial en mis experiencias [...] crearon esos momentos de aprendizaje con amor y paciencia". Juana menciona a su escuela de frontera donde manifiesta que "conocí gente increíble cuyas realidades eran muy diferentes de las mías [...] espero que sus enseñanzas me acompañen el resto de mi vida".

En las narrativas sobre los memorables de inglés en la escuela, Savannah explica que "la enseñanza es amor y pasión y mi profesora de inglés Mariela llena esos dos requisitos". En general, "los docentes de la escuela [...] sirven como ejemplos de lo que queremos y no queremos ser en el futuro" Cas evoca a su profesora de noveno porque declara que "no sólo me ayudó a ser más fluida, sino que también contribuyó a mi autoconfianza para expresarme en inglés [...] es parcialmente la razón por la cual llegué a pensar en llegar a ser una profesora". Maggie tuvo a su profesora cuatro años en la escuela. Manifiesta que "lo más impor- tante, me dio consejos desinteresados [...] Los profesores no sólo deben dar grandes clases sino también apoyar a sus estudiantes". Una profesora del instituto de Coty "rompía el hielo en la clase con su personalidad cálida".

\section{Docentes memorables del currículo universitario}

En el Profesorado de inglés donde los participantes estudian, "hay buenos profesores con un gran conocimiento del inglés [...] hay otros que no muestran su amor por lo que hacen, lo cual los estudiantes notan fácilmente" (FÁTIMA). El grupo asintió cuando Coty dijo que, además de poseer "pasión" y "entusiasmo", la mayoría de los profesores se hallan "especializados en un campo de estudio". Para ella, "ser expertos en lo que enseñamos alienta a los estudiantes a interesarse en el lenguaje". Tres profesoras mencionadas son del área de habilidades lingüísticas. Una es alguien "que realmente ama lo que enseña y lo demuestra con orgullo [...] dinámica y atrayente" (ROSE). Ant narra cómo otra docente la "alentó a seguir" proporcionando realimentación detallada. Emma explica que una docente "cuando me dio realimentación [al perder una cursada], fue amable y parecía preocupada por mí". James destaca a un profesor de fundamentos lingüísticos que enseña a través del humor planificado. Este profesor "sabía antes de la clase qué comentarios divertidos iba a hacer y precisamente cuándo iba a hacerlos" (JAMES).

Hallamos explícitamente cuatro grandes docentes del Profesorado. Clara y Tute eligen a la misma profesora porque "ha tenido un impacto tremendo en [nuestros] métodos de enseñanza y [nuestras] creencias". Se trata de una "docente sobresaliente" a causa de su "personalidad y actitud frente a sus estudiantes" (TUTE). Clara indica que sus clases "me ayudaron no sólo a mejorar mis habilidades 
escritas y orales sino también a darme cuenta de la clase de docente que querría ser en el futuro.". Marilyn escoge a una docente del área cultural pues señala que ambas "hacemos gestos cuando hablamos, ambas decimos lo que pensamos directamente y somos espontáneas [...] somos exigentes; esperamos mucho de los demás y en especial de nosotras mismas". Coty escoge a una profesora "organizada" y planificadora, que brinda realimentación positiva, "respetando a todos los estudiantes" y "creando una atmósfera acogedora".

Explorando más detalladamente en los relatos las trazas de los memorables del profesorado de inglés, observamos cómo importa la práctica moral, "la parte humana, la parte moral más que el conocimiento en sí" (RUSA). Luego, para Savannah, "un buen docente está definido por lo moral [...] cómo esta persona es como persona". Sheila valora de sus memorables que enseñen a sus estudiantes a ser buenas personas y a pensar críticamente. La pasión por la enseñanza transmitida a los estudiantes se distribuye en varias representaciones del buen docente: "pasión por la carrera" (MARILYN) y "pasión por su vocación, por la docencia" (JAMES). Sofía delibera por su propia cuenta y opina con madurez que

un buen docente [...] debe mostrar, principalmente, pasión [que] se transmitirá en sus estrategias didácticas, porque tratará de hacer que los chicos comprendan de cualquier forma, y en su personalidad, porque estará feliz y dispuesto a la hora de enseñar [...] Puede ser que un docente sea una excelente persona, agradable y súper carismática, pero si [...] no le gusta su labor, entonces toda su práctica se verá teñida de mala gana y frustración. (SOFÍA)

Respecto del costado afectivo del vínculo con los alumnos, Coty sostiene que los buenos docentes logran "establecer una buena relación" con ellos. Polka especifica que "un buen docente [...] llega a sus alumnos [...], muestra interés en que [...] comprendan y se interesen por lo que está enseñando". Fátima coincide al expresar que un buen docente hace "su trabajo con amor [...] y no como alguien 'superior'". Al indicar el conocimiento pedagógico, Jo sostiene que "un docente puede saber mucho, pero si no sabe cómo transmitirlo [...] no va a significar nada". Según Rose, "el profesor debe tener las herramientas didácticas para enseñar. Saber contenido no es sinónimo de docencia". Por otra parte, Grian revela que "en la facultad todos saben mucho, mucho [...] Hay algunos que no saben cómo manejar su conocimiento. Entonces saben que saben [...] Hay docentes que saben mucho pero no son muy pedagógicos". Juana relata que

[...] he tenido demasiados profesores [...] que son excelentes, que yo sé que son brillantes, pero [...] tienen un montón de títulos y no me transmiten nada. No entiendo lo que hablan, no se preocupan por el curso [...] Hay que hacer un énfasis, particularmente en la [universidad] nacional, [y] desarrollar todo lo que es la habilidad del docente. Eso es lo que vamos a ser. (JUANA)

Seguidamente, dos participantes tratan de hallar un equilibrio entre lo moral, lo disciplinar y lo pedagógico. De esta manera, "un docente memorable [...] presenta una combinación de buenas características que [nos] hacen admirarlo y querer seguir sus pasos" (CLARA). Según Tute, "es un combo" de lo moral y lo disciplinar que, expone, "no sólo me genera admiración sino curiosidad: 'a este profesor o profesora le puedo preguntar'”. Es un "equilibrio" que implica tener "conocimientos de los temas [...], interés personal por enseñar y aprender constantemente, [también] paciencia, ponerse en el lugar del otro [...], estar dispuesto a seguir investigando" (CAS). Finalmente, Married indica que las cualidades versátiles del "profesor ideal' varían según los contextos. Esto se debe al hecho de que

[...] en el colegio, profesores memorables eran aquellos que eran gamba, copados. En la uni- 
versidad los profesores memorables son los que van muy bien en la parte disciplinar como en el trato con los alumnos, que tienen mucha experiencia para compartir y que te pueden ayudar a formar como humano. Tal vez el profesor modelo en los institutos de inglés [...] es algo desestructurado, que sabe mucho del idioma, que ha viajado. (MARRIED)

Se trata de una disquisición muy enriquecedora que beneficia el cierre de estas comparaciones.

\section{Discusión}

Retomamos aquí brevemente nuestro interrogante acerca de cómo se entraman las narraciones estudiantiles respecto de las socialidades curriculares que habitan sus relatos. Éstas se colmaron de co-protagonistas y co-autores, tales como libros, películas, series, música, 'héroes', familiares, allegados, docentes memorables. Constituyen un verdadero menú psico-literario-cultural-social-familiar, lleno de propósitos y direcciones vitales con héroes 'reales' que señalan caminos (MCADAMS, 2006).

A la luz de nuestras tematizaciones narrativas, sostenemos que, al momento de diseñar y enseñar el currículo, en este caso universitario, se tensionan "diversos mecanismos de negociación e imposición" (DE ALBA, 1998, p. 59-60), los primeros capaces de realizar transacciones de significado y los segundos capaces de imponer o de tolerar sentidos y acciones. Las dos esferas curriculares a las que aludimos en este trabajo, la vital y la académica-formal, instituyen un mundo relacional complejo. Los estudiantes se auto-posicionan frente al currículo prescrito para su formación docente mediante sus lecturas; sus films; su música; sus héroes o sus grandes, buenos, docentes.

El currículo de las vidas (Huber et al, 2014) indudablemente aporta fondos de conocimiento para una carrera universitaria (BAUMAN, 2009; GROSSMAN y MCDONALD, 2008; SA-
RASON, 1993). Allí moran textos y representaciones literarias, televisivas, cinematográficas y musicales, así como personajes 'famosos', familiares y otros allegados (algunos de los cuales se vinculan con el currículo académico). Destacamos las épicas redentoras y agenciales narradas que pueblan este currículo vital en una simbiosis realimentada por cada estudiante al narrarlas, y potenciadas en las interacciones grupales que tuvieron lugar al contar los relatos en el aula (MCADAMS y PALS, 2006). Así, los héroes escogidos "pudieron superar circunstancias terribles" (UNNAMED). Los participantes los rescatan como mentores en una carrera que les resulta muy ardua.

Respecto del currículo formal (CAMILLONI, 2013), las historias de aprendizaje refirieron más a los docentes que a los propios partícipes-narradores y fueron, en ese sentido, relatos sobre enseñanza. Vemos que cada currículo formal "está poblado, superpoblado, con las intenciones" de estos docentes que lo co-protagonizaron (BAKHTIN, 1994, p. 293-294). La estructura profunda de las mejores maneras de enseñar conocimientos y la estructura implícita de los aspectos morales de la docencia no parecieran conjugarse en los memorables que recogimos (SHULMAN, 2005). Algunos evidencian la moralidad en sus prácticas (JACKSON, BOOSTROM y HANSEN, 2003) mientras que otros revelan sólo la pasión por enseñar (DAY, 2004). El conocimiento pedagógico de los contenidos y el curricular (SHULMAN, 1986) figuran en algunos relatos. Otros pocos docentes crearon un "entorno para el aprendizaje crítico natural" (BAIN, 2007, p. 29), diseñando problemas y tareas centrales desafiantes que atañen a la vida real.

El currículo de las vidas y el formal comportan muchas veces historias personales de transformación, de redención, de desarrollo, de generosidad y de final feliz. Son, nuevamente, variaciones de temas heroicos de indi- 
viduos solitarios que a menudo señalan el camino de la buena docencia (MCADAMS, 2006). A pesar de existir en socialidades, estos fondos de conocimiento vividos fuera de la institución escolar, así como dentro de ellas en el transcurso de la prolongada escolarización, reforzarían el valor de la intuición, de lo personal y de la supuesta relación directa entre la enseñanza y el aprendizaje (BRITZMAN, 2003).

Existen investigaciones que sugieren que los estudiantes de profesorado ya saben qué significa ser, y devenir, un buen profesor (FURLONG, 2013) desde el noviciado de la observación (LORTIE, 1975) con su currículo oculto y sus enseñanzas implícitas (JACKSON, 1996/1968, 1999). Estas conceptualizaciones niegan el sentido deficitario que la formación inicial universitaria deposita a veces sobre las experiencias previas y extramuros de los estudiantes (futuros docentes) negando el potencial de otros ámbitos para la formación - como el noviciado y la vida misma fuera de la escuela o la universidad (DAVINI, 2015). Estos espacios han sido proveedores de yacimientos (BAUMAN, 2009), fondos de conocimientos (GROSSMAN y MCDONALD, 2008), capitales narrativos (GOODSON, 2012) y, reiteradamente, currículos vitales que necesitamos recuperar a la hora de concebir planes de estudio.

Un artículo titulado "La identidad y un modelo de inversión en la lingüística aplicada" (DARVIN y NORTON, 2015) argumenta que los estudiantes necesitan saber que sus capitales culturales, sociales, lingüísticos y narrativos (BOURDIEU, 2003; GOODSON, 2012) - sus fondos de conocimiento (GROSSMAN y MCDONALD, 2008), sus yacimientos (BAUMAN, 2009) o los currículos de sus vidas (HUBER ET AL, 2014b) - exhiben potenciales significativos para su aprendizaje. Así, "la valoración de su capital es una afirmación de su identidad, una legitimación de su propio lugar en diferentes contextos de aprendizaje". Empero, puede su- ceder que "las estructuras de poder no asignen valor simbólico a [los] capital[es] que poseen" los estudiantes o que los "patrones de control" (DARVIN y NORTON, 2015, p. 10) de esas estructuras dificulten su tránsito curricular. Se torna necesario, entonces, redimir narrativamente dichos capitales para articularlos y potenciarlos en el diseño e implementación de los currículos universitarios.

\section{Conclusiones}

Los aportes de nuestra investigación al currículo de la formación inicial del profesorado universitario sugieren que los estudiantes detentan grandes caudales experienciales vivenciados, a los cuales englobamos reiteradamente bajo la categoría de aprendidos en el transcurso de un currículo de vidas transitado en paralelo a un currículo formal. En ese sentido, nuestras conceptualizaciones acuerdan con los principios de la indagación narrativa (CLANDININ, 2013), que aboga por la inclusión de un sinnúmero de historias individuales y grupales en el currículo para, en nuestro caso, la formación docente. Por eso, decimos que las narrativas de los estudiantes exigen y merecen nuestra atención ontológica para con sus existencias en devenir; nuestro celo y respeto epistemológicos por el conocimiento experiencial y vital que emerge de esos relatos; $y$ nuestra preocupación académica y profesional apoyada en el significado moral y práxico de la formación docente universitaria. Al respecto, podemos indicar que

[...] si sostenemos que la documentación narrativa ayuda a pensar teóricamente, es [...] tratar de hacer explícitos los saberes prácticos construidos al mismo tiempo que se despliegan las experiencias, para tornarlos pasibles de reflexiones, discusiones y reformulaciones. No hay práctica, ni siquiera la espontánea, que no implique ciertos saberes y supuestos acerca de la gente, su aprendizaje, sus visiones de lo 
justo, de lo deseable y de lo posible. (SUÁREZ, 2005, p. 12)

Acordando con la cita, también legitimamos al conocimiento práctico personal (CONNELLY y CLANDININ, 1988) de estos docentes en formación cuando narran acerca de los co-autores y co-protagonistas de sus currículos de vidas y de sus currículos escolares. Debemos considerar que los horizontes de este conocimiento desbordan los límites del currículo del profesorado y las fronteras de la unidad académica donde se desenvuelven. Mediante dicha legitimización, podemos entonces ampliar los confines de la formación inicial del profesorado, trasladándola fuera de las restricciones artificiales de la mera presencialidad áulica o los procesos administrativos de gestión y administración curricular.

Asimismo, cuando sostenemos que las vidas de los participantes constituyen relatos vividos al narrarse (CLANDININ, 2013), discurrimos sobre la educación en términos de un proceso de crecimiento y auto-conocimiento mediado por los relatos, promoviendo la adquisición de capitales narrativos que rupturizan los transcursos de mera reproducción social (GOODSON, 2012) en la formación del profesorado. De esta manera, el conocimiento narrativo nos abre puertas hermenéuticas para (re) interpretar los procesos de diseño del currículo y su enseñanza (GADAMER, 2004). Igualmente, sostenemos que la formación docente es una conversación permanente entre, y con, todas estas historias. Por eso, la implementación de una pedagogía narrativa (GOODSON ET AL, 2010) en los procesos de desarrollo curricular permitiría vivir, contar, volver a contar y volver a vivir relatos de enseñanza y de aprendizaje tanto dentro como fuera de la universidad (HUBER ET AL, 2014b).

Finalmente, la validez catalítica de una investigación cualitativa (GUBA y LINCOLN, 2005) refiere al grado de transformación en el cual ésta reorienta, focaliza y energiza a sus participantes, quienes se tornan activos creadores de sus vidas y contextos. La validez catalítica también ostenta implicancias políticas, justamente por el conocimiento la creatividad que genera. De esta manera, en este trabajo, hemos abandonado la matriz reproductora de la formación del profesorado y colocado a los (futuros) docentes en términos de productores y creadores del currículo a partir de sus vidas. Este planteo político sobre la valoración pública de lo privado - otorgándole atención teórica y cuidado ontológico y epistemológico - también constituye un compromiso ético con el respeto hacia todas estas dimensiones medulares, aunque poco conocidas, que son necesarias para pensar, negociar y enseñar el currículo universitario para la formación docente.

\section{Referencias}

AUSTEN, Jane. Emma. Calgary: Broadview Press, 1964.

AUSTEN, Jane. Orgullo y prejuicio. Barcelona: Austral, 2006.

BAIN, Ken. Lo que hacen los mejores profesores universitarios. Valencia: Universidad de Valencia, 2007.

BAKHTIN, Mikhail Mikhălovich. The dialogic imagination. Four essays. Austin: University of Texas Press, 1994.

BAUMAN, Zygmunt. Education in the liquid-modern setting. Power and education, Thousand Oaks, CA, SAGE, v. 1, n. 2, p. 157-166, june. 2009.

BENIGNI, Roberto. La vida es bella. Roma: Melampo Cinematografica, 1997.

BLUTH, Don; GOLDMAN, Gary. Anastasia. Burbank, CA: Walt Disney Animation Studios, 1997.

BOURDIEU, Pierre. Capital cultural, escuela y espacio social. Buenos Aires: Siglo XXI, 2003.

BRITZMAN, Deborah P; PITT, Alice. Pedagogy and 
transference: Casting the past of learning into the presence of teaching. Theory into practice, London, Taylor and Francis, v. 35, n. 2, p. 117-123, Spring. 1996.

BRITZMAN, Deborah P. Practice makes practice. A critical study of learning to teach. Revised edition. New York: State University of New York Press, 2003.

CAINE, Vera; STEEVES Pam. Imagining and playfulness in narrative inquiry. International journal of education and the arts, Philadelphia, Penn State, $v$. 10, n. 25, p. 1-14, sep. 2009.

CHBOSKY, Stephen. Las ventajas de ser invisible. Buenos Aires: Alfaguara, 2013.

CAMILLONI, Alicia. La inclusión de la educación experiencial en el currículo universitario. En: MENÉNDEZ, Gustavo. Integración docencia y extensión. Otra forma de enseñar y de aprender. Santa Fe: Universidad Nacional del Litoral, 2013. p. 11-21.

CLANDININ, D. Jean. Engaging in narrative inquiry. London-New York: Routledge, 2013.

CLANDININ, D. Jean.; MURPHY, M. Shaun. Comments on Coulter and Smith: Relational ontological commitments in narrative research. Educational researcher, Thousand Oaks, CA, SAGE, v. 38, n. 8, p. 598-602, apr. 2009.

CLANDININ, D. Jean; STEEVES, Pam; CHUNG, Simmee. Creando espacios de investigación narrativa en la formación del profesorado. En: PORTA, Luis; SARASA, María Cristina. Formación y desarrollo de la profesión docente en el profesorado: Las buenas prácticas y sus narrativas. Mar del Plata: Universidad Nacional de Mar del Plata, 2008. p. 59-83.

COLE, Ardra. The denial of the personal, the preservation of the status quo in teacher education. Journal of teaching and learning, Windsor, Ontario, University of Windsor, v. 2, n. 2, p. 1-12, dec. 2003.

COLE, Ardra; KNOWLES, J. Gary. Teacher development partnership research: A focus on methods and issues. American educational research journal, Thousand Oaks, CA, SAGE, v. 30, n. 3, p. 473-495, jan. 1993.

COLLAR, Enrique. Novena. Asunción, Paraguay: Areachika/Cine, 2012.
CONNELLY, F. Michael; CLANDININ, D. Jean. Teachers as curriculum planners: Narratives of experience. New York: Teachers College Press, 1988.

CONNELLY, F. Michael; CLANDININ, D. Jean. Narrative inquiry. In: GREEN, Judith L.; CAMILLI, Gregory; ELMORE, Patricia B. Handbook of complementary methods in education research. Mahwah, NJ: Lawrence Erlbaum, 2006. p. 477-487.

CORTÁZAR, Julio. Continuidad de los parques. En: CORTÁZAR, Julio. Final del juego. Buenos Aires: Alfaguara, 1971. p. 9-12.

CORTAZZI, Martin. Narrative analysis in ethnography. In: ATKINSON, Paul et al. Handbook of ethnography. London: SAGE, 2001. p. 384-395.

COWELL, Cressida. Cómo entrenar a tu dragón. Glendale, CA: DreamWorks, 2010.

CRANE, David; KAUFFMAN, Marta. Friends. Sherman Oaks, CA: Warner Bros. Television, 1994-2004.

CRESWELL, John W. Educational research. Planning, conducting and evaluating quantitative and qualitative research. Boston: Pearson, 2012.

CRICHTON, Michael. ER Emergencias. Nueva York: NBC, 1984-2009.

DAVINI, María Cristina. La formación en la práctica docente. Buenos Aires: Paidós, 2015.

DARVIN, Ron; NORTON, Bonny. Identity and a model of investment in applied linguistics. Annual review of applied linguistics, Cambridge, UK, CUP, v. 35, p. 36-56, March. 2015.

DAVIES, Russell T. Doctor Misterio. Londres: BBC, 2005-2018.

DAY, Christopher. A passion for teaching. London: Routledge Falmer, 2004.

DE ALBA, A. Curriculum: Crisis, mito y perspectivas. Buenos Aires: Miño y Dávila, 1998.

DEKNIGHT, Steven S. Espartaco sangre y arena. Englewood, CO: Starz, 2010.

DENZIN, Norman. K.; LINCOLN, Yvonna S. Introduction. The discipline and practice of qualitative re- 
search. In: DENZIN, Norman. K.; LINCOLN, Yvonna S. The SAGE handbook of qualitative research. Third edition. Thousand Oaks, CA: SAGE, 2005. p. 1-32.

DERRIDA, Jacques. El monolingüismo del otro o la prótesis de origen. Buenos Aires: Manantial, 1996.

DEWEY, John. Experience and nature. London: George Allen y Unwin, 1929/1925.

FALCONES, Ildefonso. La mano de Fátima. México: Grijalbo, 2010.

FELLOWES, Julian. La joven Victoria. Santa Mónica, CA: GK Films, 2009.

FLYNN, Gillian. Perdida. Milan: Mondadori, 2013.

FOLLETT, Ken. Los pilares de la tierra. Barcelona: Plaza \& Janés, 2017.

FRANK, Anne. Diario de Ana Frank. Buenos Aires: Hemisferio, 1958.

FRASER, Sharon P.; BOSANQUET, Agnes M. The curriculum? That's just a unit outline, isn't it? Studies in higher education, London, Taylor and Francis, v. 31, n. 3, p. 269-284, jun. 2006.

FREY, James. En mil pedazos. Buenos Aires: Taurus, 2006.

FURLONG, Catherine. The teacher I wish to be: Exploring the influence of life histories on student teacher idealised identities. European journal of teacher education, London, Taylor and Francis, v. 36, n. 1, p. 68-83, feb. 2013.

GADAMER, Hans-Georg. Truth and method. London-New York: Continuum, 2004.

GARCÍA MÁRQUEZ, Gabriel. El amor en los tiempos del cólera. Buenos Aires: Debolsillo, 2003.

GILLIGAN, Vince. Breaking bad. New York: AMC, 2008-2013.

GOODSON, Ivor F. Developing narrative theory: Life histories and personal representation. Abingdon, Oxon, UK-New York: Routledge, 2012.

GOODSON, Ivor F. ET AL. Narrative learning. Abingdon, Oxon, UK-New York: Routledge, 2010.

GROSSMAN, Pam; MCDONALD, Morva. Back to the fu- ture: Directions for research in teaching and teacher education. American educational research journal, Thousand Oaks, CA., SAGE, v. 45, n. 1, p. 184-205, mar. 2008.

GRUBER, J. Mackye; BRESS, Eric. El efecto mariposa. Los Angeles: BenderSpink, 2004.

GUBA, Egon G.; LINCOLN, Yvonna S. Paradigmatic controversies, contradictions, and emerging confluences. In: DENZIN, Norman. K.; LINCOLN, Yvonna $S$. The SAGE handbook of qualitative research. Third edition. Thousand Oaks, CA: SAGE, 2005. p. 191-215.

GUILLEMIN, Marilys; GILLAM, Lynn. Ethics, reflexivity, and "ethically important moments" in research. Qualitative inquiry, Thousand Oaks, CA., SAGE, v. 10, n. 2, p. 261-280, apr. 2004.

HERNÁNDEZ, José. Martín Fierro. Buenos Aires: Ediciones Lea, 2017.

HOMERO. La Iliada. Buenos Aires: Gradifco, 2006.

HÖKKÄ, Päivi; ETELÄPELTO, Anneli; RASKU-PUTTONEN, Helena. Recent tensions and challenges in teacher education as manifested in curriculum discourse. Teaching and teacher education, Atlanta, GA, Elsevier, v. 26, n. 4, p. 845-853, may. 2010.

HUBER, Janice ET AL. Shifting stories to live by: Teacher education as a curriculum of narrative inquiry identity explorations, Reflective practice, London, Routledge, v. 15, n. 2, p.176-89. mar. 2014 a.

HUBER, Janice ET AL. La indagación narrativa como pedagogía en la educación: el potencial extraordinario de vivir, contar, volver a contar y revivir relatos de experiencias, Revista de Educación, Mar del Plata, Universidad Nacional de Mar del Plata, v. 5, n. 7, p. 33-74, ago. 2014b.

JACKSON, Philip W. La vida en las aulas. Nueva introducción del autor. Madrid: Paideia-Morata, $1996 / 1968$.

JACKSON, Philip W. Enseñanzas implícitas. Buenos Aires: Amorrortu, 1999.

JACKSON, Philip W.; BOOSTROM, Robert E.; HANSEN, David T. La vida moral en la escuela. Buenos Aires: Amorrortu, 2003. 
JOYCE, James. Retrato del artista adolescente. México: Terramar Ediciones, 2016.

KRIPKE, Eric. Supernatural. Burbank, CA: Warner, 2008-2009.

LEARY, Denis; TOLAN, Peter. Rescátame. Culver City, CA: Sony Pictures Television, 2004-2011.

LEONCAVALLO, Ruggero. Payasos. Milán, 1892.

LONDON, Jack. White Fang. London: Penguin, 2008.

LORTIE, Dan C. Schoolteacher: A sociological study. Chicago: University of Chicago Press, 1975.

MCADAMS, Dan P. Personality, modernity, and the storied self: A contemporary framework for studying persons. Psychological inquiry, London, Taylor and Francis, v. 7, n. 4, p. 295-321, oct. 1996.

MCADAMS, Dan P. The redemptive self: Generativity and the stories Americans live by. Research in human development, London, Taylor and Francis, v. 3, n. 2-3, p. 81-100, sep. 2006.

MCADAMS, Dan P. The life story interview. Evanston, IL: Northwestern University, 2008.

MCADAMS, Dan P. Life authorship: A psychological challenge for emerging adulthood, as illustrated in two notable case studies. Emerging adulthood, Thousand Oaks, CA., SAGE, v. 1, n. 2, p. 151-158, jun. 2013.

MCADAMS, Dan P.; HANEK, Kathrin J.; DADABO, Joseph G. Themes of self-regulation and self-exploration in the life stories of religious American conservatives and liberals. Political psychology, Hoboken, NJ, John Wiley \& Sons, v. 34, n. 2, p. 201-219, apr. 2013.

MCADAMS, Dan P.; PALS, Jennifer L. A new Big Five: Fundamental principles for an integrative science of personality. American psychologist, Washington DC, APA, v. 61, n. 3, p. 204-217, apr. 2006.

MCCULLOUGH, Collen. El pájaro canta hasta morir. Barcelona: Plaza \& Janés, 1995.

MCEWAN, Ian. Expiación. Barcelona: Anagrama, 2008.

MEENAGHAN, Jim. Persiguiendo mavericks. Los Ángeles: Fox 2000 Pictures, 2012.
MEYER, Stephenie. Crepúsculo. Buenos Aires: Alfaguara, 2015.

MILNE, Alan Alexander. Mis cuentos de Winnie the Pooh. Madrid: Cegal, 2008.

MITCHELL, Dominic. In the flesh. London: BBC, 20132015.

MOGGACH, Deborah. Orgullo y prejuicio. Nueva York: Focus Features, 2005.

NORTON, Bonny. Identity and language learning. Extending the conversation. Second edition. Bristol-Buffalo-Toronto: Multilingual Matters, 2013.

ORCI, Roberto; KURTZMAN, Alex. Transformers. Glendale, CA: DreamWorks, 2007.

POPE, Rob. Textual intervention. London: Routledge, 1995.

QUIROGA, Horacio. A la deriva. En: QUIROGA, Horacio. A la deriva y otros cuentos. Buenos Aires: Colihue, 1998. p. 51-55.

RHIMES, Shonda. Anatomía de Grey. Nueva York: ABC, 2005-2017.

RICHARDSON, Laurel; ST. PIERRE, Elizabeth Adams. Writing. A method of inquiry. In: DENZIN, Norman K.; LINCOLN, Yvonna S. The SAGE handbook of qualitative research. Third edition. Thousand Oaks, CA: SAGE, 2005. p. 959-978.

ROTH, Eric. Forrest Gump. Hollywood, CA: Paramount, 1994.

ROWLING, Joanne K. Harry Potter y la piedra filosofal. Barcelona: Emecé, 1999.

SANDLIN, Jennifer A.; SCHULTZ, Brian D.; BURDICK, Jake. Handbook of public pedagogy: Education and learning beyond schooling. New York-London: Routledge, 2010.

SANTA ANA, Antonio. Los ojos del perro siberiano. Buenos Aires: Norma, 1998.

SARASON, Seymour B. The case for change: Rethinking the preparation of educators. San Francisco, CA: Jossey-Bass, 1993.

SCORSESE, Martin. Toro Salvaje. Culver, CA: Charto- 
ff-Winkler Productions, 1980.

SETTERFIELD, Diane. El cuento número trece. Buenos Aires: Lumen, 2007.

SHEPARD, Sara. Pequeñas mentirosas. Chattanooga, TN: ABC Family, 2010-2016.

SHULMAN, Lee S. Those who understand: knowledge growth in teaching. Educational researcher, Thousand Oaks, CA., SAGE, v. 15, n. 2, p. 4-14, feb. 1986.

SHULMAN, Lee S. Signature pedagogies in the professions. Daedalus, Cambridge MA, MIT Press, v. 134, n. 3, p. 52-59, Summer. 2005.

SPARKS, Nicholas. Dear John. New York: Warner Books, 2007.

ST. CLAIR ROBSON, Lucia. Ride the wind. New York: Random House, 1982.

SUÁREZ, Daniel. La documentación narrativa de experiencias pedagógicas. Una estrategia para la formación de docentes. Buenos Aires: Ministerio de Educación, Ciencia y Tecnología-AICID, 2005.
VAN MANEN, Max. Investigación educativa y experiencia vivida. Barcelona: Idea Books, 2003.

VON GOETHE, Johann Wolfgang. Las penas del joven Werther. Barcelona: Alba Clásica, 2011.

TAYLOR, Carol A.; BOVILL, Catherine. Towards an ecology of participation: Process philosophy and co-creation of higher education curricula. European educational research journal, Thousand Oaks, CA., SAGE, 1474904117704102 , p. 1-17, apr. 2017.

TOLKIEN, John Ronald Reuel. El señor de los anillos. Barcelona: Minotauro, 2001.

TORNATORE, Giuseppe. Cinema Paradiso. Roma: Cristaldifilm, 1988.

TREDWELL-OWEN, Caspian. La isla. Glendale, CA: DreamWorks, 2005.

WOOLF, Virginia. La señora Dalloway. Tres Cantos, España: Akal, 2017.

Recebido em: 22.12.2017 Aprovado em: 30.03.2018

María Cristina Sarasa Profesora Titular Regular de la cátedra de Comunicación Integral, Universidad Nacional de Mar del Plata. Departamento de Lenguas Modernas. Investigadora del Grupo de Investigaciones en Educación y Estudios Culturales, Centro de Investigaciones Multidisciplinarias en Educación (CIMEd). e-mail: mcsarasa@hotmail.com

Funes 3350- Teléfono: 542234752277 